\title{
A Magnetometer Calibration Method Using Single-Axis Motion Trajectory and Unscented Kalman Filter for Body Motion Capture Applications
}

\author{
Farzan Farhangian $^{1,}$, , Saoussen Bilel ${ }^{1}$, Faramarz Farhangian ${ }^{2}$, Rene Jr. Landry ${ }^{1}$ \\ ${ }^{1}$ Department of Electrical Engineering, ETS University of Quebec, Montreal, Canada \\ ${ }^{2}$ Department of Statistics, Shahid Beheshti University, Tehran, Iran \\ Email address: \\ Farzan.farhangian@lassena.etsmtl.ca (F. Farhangian), Saoussen.bilel@lassena.etsmtl.ca (S. Bilel), Renejr.landry@etsmtl.ca (R. Jr. Landry) \\ ${ }^{*}$ Corresponding author
}

To cite this article:

Farzan Farhangian, Saoussen Bilel, Faramarz Farhangian, Rene Jr. Landry. A Magnetometer Calibration Method Using Single-Axis Motion Trajectory and Unscented Kalman Filter for Body Motion Capture Applications. International Journal of Sensors and Sensor Networks. Vol. 9, No. 1, 2021, pp. 1-10. doi: 10.11648/j.ijssn.20210901.11

Received: December 18, 2020; Accepted: December 25, 2020; Published: January 12, 2021

\begin{abstract}
Magnetometer calibration is a pre-processing step in the Attitude and Heading Reference Systems (AHRS) which has an essential role in many applications. The main purpose of this article is to derive an innovative and precise calibration approach for a magnetometer set installed on human body. To execute this calibration method, all the error parameters of multiple magnetometers are considered in an Unscented Kalman Filter (UKF) model for accurate estimation of calibration parameters. As achieving a precise estimation in Kalman filters-based algorithms needs an accurate and complete observation model, a special single-axis rotation trajectory for Inertial Measurement Unit (IMU) is performed to increase the observability rank of the calibration model. To evaluate the proposed method, five body-mounted sensors were experimented in the laboratory at the same time for applying in the body motion capture system. The results showed that all five sensors were well-calibrated without any need to be detached from the body and using any rotational robot arm. The resolution and precision of the proposed calibration method are assessed by the ellipsoid-fitting representation method. Consequently, all the body-mounted magnetometers were calibrated, on average, by about $1 \%$ uncertainty. The method can be used in every motion capture and AHRS applications due to its feasibility and simplicity.
\end{abstract}

Keywords: Magnetometer Calibration, Calibration, Motion Capture, Magnetometer, Inertial Sensors, Unscented Kalman Filter, MEMS

\section{Introduction}

With prompt advancements in technology, the development of Microelectromechanical Systems (MEMS) has been under deep investigation of researchers and industries. The integrated MEMS technology has had underlying impacts in various applications like Inertial Navigation Systems (INS), positioning and guidance, body motion capture, Attitude and Heading Reference Systems (AHRS), object tracking, etc. The magnetic field can be considered as an important measurement in Kalman Filter (KF)-based AHRS [1, 2], INS, and motion capture models [3], also, some important attitude determination methods utilize the magnetometer as a compass to reduce the heading drift in Heuristic Drift Reduction (HDR) algorithm [4]. The mentioned systems are usually implemented using 9 Degrees of Freedom (DoF) inertial and magnetic sensors, namely, three-axis accelerometer, three-axis gyroscope, and three-axis magnetometers. The long-term accuracy of these systems is partially based on the system's calibration. Although many methods aim to calibrate the bias and other error parameters of inertial gyroscope and accelerometers onboard, calibration of magnetometers is dependent on various parameters which made it more challenging. Calibration of magnetometer pertains to various parameters and error sources like bias, misalignment error, soft and hard iron effects, and the scale factor. The hard-iron effect is usually caused by the materials which generate a constant offset to each magnetometer axis. This constant 
distortion depends on the position and orientation of the material relative to the sensor. However, the soft-iron effect is not necessarily constant which means, it can be compensated with more difficult procedures. Moreover, the soft-iron distortion is related to the attitude of the material relative to the magnetic field, and the sensors [1].

Orientation estimation and especially, the heading needs accurate magnetometer data, with respect to the fact that the heading is strongly vulnerable to magnetic disturbances. Although obtaining precise magnetometer data depends on the environment situation, presenting the suitable sensor's model has a significant impact on the calibration results. Apart from that, the calibration precision is under effect of temperature and pressure, especially in spacecraft and aerospace utilizations. In the class of environmental disturbance, the hard iron disturbances are usually caused existence of ferromagnetic objects and materials with continuous magnetic fields, on the other hand, the soft iron perturbations are commonly affected by the variation of surrounding magnetic fields. Most of the magnetometer calibration methods are based on batch methods in which the magnetometer's data is recorded to process and obtain the calibration parameters. Also, some methods have utilized the motion trajectory for the magnetometer which needs each sensor to be rotated in the same trajectory. These methods need to implement the rotation trajectory for all the sensors one by one, especially in body motion capture applications that the mounted sensors are in various coordinate and axis locations. In the traditional methods, for following the trajectory, each sensor should be rotated around its $\mathrm{x}, \mathrm{y}$, or $\mathrm{z}$-axis, while all the sensors are mounted on the body and implementing these trajectories are consuming the time, energy, and detachments. In this paper, the new method is presenting using single axis motion trajectory and UKF model which is not dependent on the attitude of the sensor's coordinates. The error parameters of all the body-mounted sensors can be estimated at the same time with acceptable accuracy.

In Section 2, we present an overview of previous works and papers, relevant to our research. In Section 3, we presented a model for the error of one magnetometer set which consists of five magnetometer sensors mounted on the body, also, the UKF parameters and update stages are discussed completely. The details of the proposed method, rotation trajectory, and observability analysis are described in section 4. The experiment, results, and statistical analysis are presented in Section 5 and finally, the conclusions from the paper and future directions are presented in Section 6.

\section{Related Works}

Precise output data from nine Degrees of Freedom (DoF) Inertial Measurement Unit (IMU) comprised of 3-axis accelerometer, 3 -axis gyroscope, and 3 -axis magnetometer, is needed in many applications like Inertial Navigation Systems (INS), Attitude and Heading Reference Systems (AHRS), missile guidance, body motion tracking, and spacecraft. Apart from the importance of the accelerometer and the gyroscope calibrations, magnetometer calibration has a particular impact on the result of mentioned systems due to its impressionability from kinds of magnetic distortion. Besides bias, scale factor, and nonorthogonality parameters which are common in all inertial sensors, magnetometer calibration requires the estimation of soft and hard iron effects. With investigating the papers and previous works about magnetometer calibration, most of the methods can be categorized in Kalman-based algorithms, Least Square (LS) Solutions, Maximum Likelihood estimators (ML), optimization approaches, and IMU rotation methods.

A nonlinear least square and simple batch LS method has experimented, which demonstrated the reduction in standard deviation $[5,6]$. Also, in parallel, the significant effort was done in regular ML filters and estimators with quartic subjective function [7], and ML calibration with combination of sensors [8]. A swarm optimization algorithm was performed in some works which are more likely to be used in spacecraft application due to its independency to alignment procedure [9]. Many endeavors were accomplished also in Kalman-based calibration algorithms in which linear or nonlinear sensor models were designed for estimating the calibration parameters. Calibration with Kalman Filter (KF) and covariance matrices [10], Extended Kalman Filter (EKF) designs [11], the combination of EKF with semi-random motion sequence [12], adding the gyroscope's output as observation in EKF [13], and Unscented Kalman Filter (UKF)-based calibration system [14] showed that in some cases, EKF can be more precise method due to its compatibility and reliability in many applications. However, in this paper, a novel observability analysis method and an IMU rotation technique aided a UKF estimator with extremely nonlinear measurement model. The defined states of the UKF system have been estimated with higher observability rank after performing the rotation technique. Apart from that, this method presents a precise estimation of calibration parameters with single rotation for all IMUs mounted on the body, which prevent to perform the rotation for the IMUs, one by one.

Another algorithm category utilizes IMU rotation with both pre-defined or unknown trajectory which all of them since now need turntable or rotatory platform for rotating the IMU in an exact defined angle which is the main disadvantage of them as it is unacceptable for applications like motion capture that prefer to perform the calibration on body-mounted sensors. Calibration using 3-axis rotation platform [15], and 1 -axis rotation [16] are designed and examined. Also, there were some efforts in magnetometer calibration with estimating the calibration nonlinearity coefficients [17], however; one of the most reliable and practicable calibration methods is calibrating using the ellipsoid fitting algorithm, in which fitting the calibrated data of magnetometer with unit ellipsoid shape has been the evaluating parameter for calibration's accuracy [18]. The Triad and UKF algorithms are investigated for real-time magnetometer calibration and attitude estimation [19]. However, these algorithms presented an accurate orientation results only for the satellite applications and it can not be extended to various demands. A 
great effort has been shown on accuracy improvement of heading using calibrated data of magnetometer in real-time [20]. Their design has demonstrated two degrees reduction in residual magnetic heading error; however, the system did not study with the uncertainty of calibration parameters. Some novel calibration methods like utilizing BP neural network and artificial data are considered in recent years [21, 22]. In this paper, the magnetometer sensors are modeled for UKF to estimate the calibration parameters and the single rotation trajectory is defined in any initial attitude of sensors to increase the observability rank of the calibration model. the rotation trajectory does not need any detachment and can be performed as the sensors are mounted in the body. Finally, the method tested with real low-cost 9-DoF IMUs for motion capture application.

\section{Error Model of a Magnetometer Set}

\subsection{UKF State Space Model}

The Complete model of error for a triad magnetometer of an IMU is considered in Eq. (1) [18].

$$
\widehat{m}=\mathrm{Am}+\mathrm{b}+\varepsilon
$$

In which, $\hat{m}$ and $\mathrm{m}$ are uncalibrated and calibrated magnetometer data, respectively. $\mathrm{A}$ is a three-dimensional matrix that consists of combination of the scale factor, nonorthogonality, and soft iron error parameters, also, b and $\varepsilon$ are combined bias vector and measurement noise, respectively. $\mathrm{A}$ and $\mathrm{b}$ are defined in (2) and (3) [18].

$$
\begin{gathered}
A=S M A_{S_{-} \text {iron }} \\
b=S M b_{h_{-} \text {iron }}+b_{\text {off }}
\end{gathered}
$$

The $S$, and $M$ are the scale factor and the nonorthogonality matrices, respectively, Also, the parameters $A_{s_{\text {iniron }}}, b_{h_{-} \text {iron }}$ and $b_{o f f}$ are 3 by 3 soft iron effect matrix, hard iron effect vector, and the sensor's offset, respectively [18]. The complete calibration can be performed with the estimation of bias and $\mathrm{A}$ matrix due to the calibration equation in Eqs. (4) [18].

$$
m=A^{-1}(\widehat{m}-b)
$$

Because the magnitude of the true local magnetic field in the same location should be constant, the given norm in the Eq. (5) should be a constant value. Therefore, the $\left(A^{-1}\right)^{T} A^{-1}$, and consequently, $A$ must be symmetric matrices that the Eq. (5) be considered as an ellipse with center of $b$ and a tilt shape originated from the A [23].

$$
\begin{gathered}
\|m\|^{2}=m^{T} m=\left(A^{-1}(\widehat{m}-b)\right)^{T} A^{-1}(\widehat{m}-b) \\
=(\widehat{m}-b)^{T}\left(A^{-1}\right)^{T} A^{-1}(\widehat{m}-b) \\
A=\left[\begin{array}{lll}
a_{11} & a_{12} & a_{13} \\
a_{12} & a_{22} & a_{23} \\
a_{13} & a_{23} & a_{33}
\end{array}\right]
\end{gathered}
$$

As matrix A, given in Eq. (6) is a symmetric matrix, the state vector of the UKF model is comprised of $a_{11}, a_{22}, a_{33}$, $a_{12}, a_{13}$ and $a_{23}$ as elements of A matrix, $b_{x}, b_{y}$ and $b_{z}$ as elements of bias vector and $m_{x}, m_{y}$ and $m_{z}$ as calibrated magnetometer estimations. Eqs. (7-10) demonstrate the state transition model of UKF for a magnetometer set included $n$ magnetometers [23]. The total states of the system are $12 \mathrm{n}$ consists of 3 states for calibrated magnetometer data, 6 states for elements of matrix A and 3 states for bias for each sensor.

$$
\begin{gathered}
x_{k+1}=F_{k} x_{k}+v_{k}, \\
x_{k}=\left[m_{1}, \cdots, m_{n}, A_{1}, \cdots, A_{n}, b_{1}, \cdots, b_{n}\right]^{T} \\
F_{k}=\left[\begin{array}{cc}
\Sigma_{k} & 0_{3 n \times 9 n} \\
09 n \times 3 n & I_{9 n \times 9 n}
\end{array}\right] \\
\Sigma_{k}=\left[\begin{array}{ccc}
G_{k}^{1} & 0 & 0 \\
0 & \ddots & 0 \\
0 & 0 & G_{k}^{n}
\end{array}\right] \\
G_{k}^{n}=\left[\begin{array}{ccc}
1 & -\omega_{z}^{n} \cdot d t & \omega_{y}^{n} \cdot d t \\
\omega_{z}^{n} \cdot d t & 1 & -\omega_{x}^{n} \cdot d t \\
-\omega_{y}^{n} \cdot d t & \omega_{x}^{n} \cdot d t & 1
\end{array}\right]
\end{gathered}
$$

In which, $\mathrm{n}$ is the number of sensors and $m_{n}, a_{n}$ and $b_{n}$ are the states of sensor n. $m_{n}=\left[m_{x}^{n}, m_{y}^{n}, m_{z}^{n}\right]^{T}$, in which the elements of that are calibrated magnetometer output in three axes. elements of soft iron, hard iron, and scale factor combined matrix for sensor $\mathrm{n}$ is defined as $A_{n}=\left[a_{11}^{n}, a_{22}^{n}, a_{33}^{n}, a_{12}^{n}, a_{13}^{n}, a_{23}^{n}\right]^{T}$. Moreover, $\omega_{x}^{n}, \omega_{y}^{n}$, and $\omega_{z}^{n}$ are calibrated gyroscope's outputs and elements of bias vector for sensor $\mathrm{n}$ in its three-axis is $b_{n}=\left[b_{x}^{n}, b_{y}^{n}, b_{z}^{n}\right]^{T}$. Also, $I$ is the identity matrix, and dt defined as the sampling time. The nonlinear observation model is defined as Eqs. (11-13), in which the uncalibrated magnetometer measurements are measurements of the system and $\varepsilon_{m}$ defined as a white Gaussian noise [24].

$$
\begin{gathered}
z_{k}=h\left(x_{k}\right)+\varepsilon_{m}, h\left(x_{k}\right)=\left[h_{1}\left(x_{1}^{k}\right), \cdots, h_{n}\left(x_{n}^{k}\right)\right]^{T} \\
h_{n}\left(x_{n}^{k}\right)=\left[\begin{array}{ccc}
a_{11}^{n} & a_{12}^{n} & a_{13}^{n} \\
a_{12}^{n} & a_{22}^{n} & a_{23}^{n} \\
a_{13}^{n} & a_{23}^{n} & a_{33}^{n}
\end{array}\right]\left[\begin{array}{c}
m_{x}^{n} \\
m_{y}^{n} \\
m_{z}^{n}
\end{array}\right]+\left[\begin{array}{c}
b_{x}^{n} \\
b_{y}^{n} \\
b_{z}^{n}
\end{array}\right] \\
h\left(x_{k}\right)=\left[\begin{array}{lll}
A_{1} & \ldots & A_{n}
\end{array}\right]\left[\begin{array}{c}
m_{1} \\
\vdots \\
m_{n}
\end{array}\right]+\left[\begin{array}{c}
b_{1} \\
\vdots \\
b_{n}
\end{array}\right]
\end{gathered}
$$

Where, the $h_{n}\left(x_{n}^{k}\right)$ is the measurement of the $\mathrm{n}$-th magnetometer sensor. The UKF estimation is performed for this system with a linear state transition matrix and a nonlinear observation matrix.

\subsection{UKF Updates and Parameters}

As the Unscented Kalman filter utilizes the extension form of Unscented Transformation (UT), it needs to define a new state model with original states and noise variables. At the first stage, the sigma point is obtained using the propagation of a random variable $x$ through a nonlinear function, $f(x)$. The new propagated state, $x_{k}^{a}$, is defined as Eq. (14), also, the 
corresponding sigma matrix, $X_{k}^{\prime}$ is mentioned in the Eqs. (15-17).

$$
\begin{gathered}
x_{k}^{a}=\left[\begin{array}{ccc}
x_{k}^{T} & v_{k}^{T} & n_{k}^{T}
\end{array}\right]^{T} \\
x_{k}^{a}=\bar{x} \\
x_{k}^{a}=\bar{x}+\left(\sqrt{(L+\gamma) P_{x}}\right)_{i}, i=1, \ldots, L \\
x_{k}^{a}=\bar{x}-\left(\sqrt{(L+\gamma) P_{x}}\right)_{i-L}, i=L+1, \ldots, 2 L
\end{gathered}
$$

Where, $\bar{x}$ is the mean value of a random variable, $P_{x}^{a}$ is its Covariance, $\mathrm{L}$ is the dimension of the random variable, and $\gamma$ defined as scaling parameter with value of $\beta^{2}(L+O)-L$. Herein, $\beta$ is a constant, that determines the distribution of sigma point around the mean value. $\beta$ should be usually a small value so, here it is defined as a unit value. Also, $O$ is equivalent to $3-\mathrm{L}$. Therefore, the sigma points matrix is defined is Eq. (18).

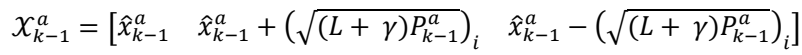

The Eqs. (19-23) demonstrate the time update section of the UKF model.

$$
\begin{gathered}
x_{k \mid k-1}^{x}=F\left[x_{k-1}^{x}, x_{k-1}^{v}\right] \\
\hat{x}_{k}^{-}=\sum_{i=0}^{2 L} W_{i}^{(m)} x_{i, k \mid k-1}^{x} \\
P_{k}^{-}=\sum_{i=0}^{2 L} W_{i}^{(c)}\left[X_{i, k \mid k-1}^{x}-\hat{x}_{k}^{-}\right]\left[x_{i, k \mid k-1}^{x}-\hat{x}_{k}^{-}\right]^{T} \\
y_{k \mid k-1}=H\left[x_{k \mid k-1}^{x}, x_{k-1}^{n}\right] \\
\hat{y}_{k}^{-}=\sum_{i=0}^{2 L} W_{i}^{(m)} y_{i, k \mid k-1}
\end{gathered}
$$

Where, $W_{i}$ are the UKF weights with values of $0.5(L+$ $\gamma)^{-1}$ for both $W_{i}^{(m)}$, and $W_{i}^{(c)}$. To update the measurement, the Kalman gain should be calculated. Eqs. (24-28) are mentioned in the measurement update stage for the UKF model.

$$
\begin{gathered}
P_{\tilde{y}_{k} \tilde{y}_{k}}=\sum_{i=0}^{2 L} W_{i}^{(c)}\left[\mathcal{Y}_{i, k \mid k-1}-\hat{y}_{k}^{-}\right]\left[\mathcal{Y}_{i, k \mid k-1}-\hat{y}_{k}^{-}\right]^{T} \\
P_{x_{k} y_{k}}=\sum_{i=0}^{2 L} W_{i}^{(c)}\left[x_{i, k \mid k-1}-\hat{x}_{k}^{-}\right]\left[\mathcal{Y}_{i, k \mid k-1}-\hat{y}_{k}^{-}\right]^{T} \\
K=P_{x_{k} y_{k}} P_{\tilde{y}_{k} \tilde{y}_{k}}{ }^{-1} \\
\hat{x}_{k}=\hat{x}_{k}^{-}+K\left(y_{k}-\hat{y}_{k}^{-}\right) \\
P_{k}=P_{k}^{-}-K P_{\tilde{y}_{k} \tilde{y}_{k}} K^{T}
\end{gathered}
$$

Here, the $\mathcal{Y}_{i, k}$ is the corresponding measurement of the UKF system in i-th row of the measurement matrix and time-step $\mathrm{k}$. Also, $\mathrm{K}$ is the Kalman gain and $\hat{x}_{k}$ is the final estimated state. To initialize the system, the initial states, initial covariance matrix, initial new propagated state vector, and the initial random variable's covariance should be determined, as Eqs. (29-32).

$$
\begin{gathered}
\hat{x}_{0}=\mathrm{E}\left[x_{0}\right] \\
P_{0}=\mathrm{E}\left[\left(x_{0}-\hat{x}_{0}\right)\left(x_{0}-\hat{x}_{0}\right)^{T}\right]
\end{gathered}
$$

$$
\begin{aligned}
& \hat{x}_{0}^{a}=E\left[x^{a}\right]=\left[\begin{array}{lll}
\hat{x}_{0}^{T} & 0 & 0
\end{array}\right]^{T} \\
& P_{0}^{a}=\mathrm{E}\left[\left(x_{0}^{a}-\hat{x}_{0}^{a}\right)\left(x_{0}^{a}-\hat{x}_{0}^{a}\right)^{T}\right]
\end{aligned}
$$

\section{Proposed Method}

The main subjective of the proposed method is rotation of several body mounted IMUs for increasing the observability rank of the system. As a result, the proper mathematical metrics should be defined for analyzing the observability of the complete system. The methods for this analysis are different before and after performing the rotation algorithm since by performing the proposed method, the problem will be changed from a discretized time-variant system to a brief time interval system and each rotation will be defined as an interval in the calibration process. The nonlinear observability analysis is considered to determine the observability rank of the system before the proposed method and the Piece-Wise Constant System (PWCS) analysis method is applied to determine the rank of observability after executing the method. For investigating nonlinear observability, the Lie derivative function is required, and by calculating the rank of Jacobian for $\Phi(x, u)$, the observability condition of the defined system will be examined. As $z_{k}$ consists of three observation in three $\mathrm{x}, \mathrm{y}$, and $\mathrm{z}$ axes, the observation function has three elements of $h_{1}, h_{2}$ and $h_{3}$. The Lie derivative equations are considered in Eqs. (33-35) [25].

$$
\begin{gathered}
\varphi_{i}^{0}=h_{i} \\
\varphi_{i}^{j}=\frac{\partial \varphi_{i}^{j-1}}{\partial x} F+\sum_{k=0}^{j-1} \frac{\partial \varphi_{i}^{j-1}}{\partial u^{(k)}} u^{(k+1)} \\
\Phi(x, u)=\left(h_{1}, \varphi_{1}^{1}, \ldots, \varphi_{i}^{k_{1}-1}, \ldots, h_{m}, \varphi_{m}^{1}, \ldots, \varphi_{m}^{k_{m}-1}\right)^{T} \\
\operatorname{rank}\left(\frac{\partial \Phi(x, u)}{\partial x}\right)=o b s
\end{gathered}
$$

The obs amount determines the observability rank of the system. For the system with n sensors, the full observability rank is $12 \mathrm{n}$ with regards to the model defined in the Eq. (7).

\subsection{PWCS Observability Analysis Method}

The Piece-Wise Constant System (PWCS) is kind of observability analysis method for linear time-variant systems that investigate the observability rank with the least amount of computation [26]. As our system is completely nonlinear, for analyzing the observability in short term rotation intervals, the measurement model of the system should be linearized with obtaining the Jacobian matrix, as Eq (36).

$$
H_{k}=\frac{\partial h(x)}{\partial x}
$$

For the system consists of $\left[F_{k} H_{k}\right]$, the observability rank in an interval of $(k, k+1)$ is the rank of stripped observability matrix (SOM) which is defined in Eqs. $(37,38)[26]$.

$$
\begin{gathered}
Q_{k}=\left[\begin{array}{llll}
H_{k}{ }^{T} & \left(H_{k} F_{k}\right)^{T} \quad\left(H_{k} F_{K}^{2}\right)^{T} \cdots & \left(H_{k} F_{K}^{n-1}\right)^{T}
\end{array}\right]^{T} \\
\operatorname{SOM}(k)=\left[\begin{array}{llll}
Q_{1} & Q_{2} & \cdots & Q_{k}
\end{array}\right]^{T}
\end{gathered}
$$




\subsection{Body Rotation Trajectory}

Five IMUs, each one consists of a 3-axis accelerometer, a 3-axis gyroscope, and a 3-axis magnetometer, considered a set of IMUs which are installed on the body. The objective of the proposed calibration method is to calibrate all the magnetometers at the same time while mounted on the body. In contrast to other rotation-based calibration methods, this method does not need any detachment from the body for performing the rotation. Figure 1 demonstrates five upper body-mounted sensors.



Figure 1. Rotation Trajectory for magnetometer set calibration of five upper body-mounted IMUs.

The rotation trajectory is performed in 7 steps (with considering the initial step) and 6 movements. The number of states with these five sensors is 60 , in total. After calculating the observability rank of the system before performing the proposed method with the nonlinear observability analysis method, the observability rank of the system obtained as 40 , which shows that the system does not have full observability rank. However, the proposed rotation trajectory was performed with five body-mounted IMUs in 7 time-steps. If $h_{n}\left(x_{n}^{k}\right)$ defined as the measurement of sensor $\mathrm{n}$ in the time-step $\mathrm{k}$, there are 7 measurements in the proposed rotation trajectory. For the sensors installed in right and left hands, the rotation is among the z-axis, and for the other sensors which mounted on the chest, the rotation is among its y-axis. The observability rank of the general system after the time-step 7 is calculated as SOM matrix which obtained from $H_{1}, \ldots, H_{7}$, as Eq. (39).

$$
\operatorname{SOM}(7)=\left[\begin{array}{cccc}
H_{1} & H_{2} & & H_{7} \\
H_{1} F_{1} & H_{2} F_{2} & & H_{7} F_{7} \\
\vdots & \vdots & \cdots & \vdots \\
H_{1} F_{1}^{11} & H_{2} F_{2}^{11} & & H_{7} F_{7}^{11}
\end{array}\right]^{T}
$$

The rank of the mentioned SOM matrix after the proposed 7-step rotation trajectory was calculated as 57 observable states. Although the observable states are not still full rank, the proposed method has shown the improvement in state observation of the system, which can lead to progress the inaccuracy of state estimation is the UKF system.

\section{Experimental Evaluation}

The experiment was performed with five MEMS-based 9-DoF IMUs mounted on the torso, right and left upper arm, right and left forearm. The environment of the experiment was in a laboratory full of electronic equipment and iron materials, which leads to providing a situation of medium-level magnetic disturbance for each sensor. To perform the rotation method, no precise rotational platform was used in the experiment, and the rotations were performed only with a degree rated surface plate on the ground which was marked in $\pm 90^{\circ}$. Using no precise rotation platform was because of validating the experiment with accuracy uncertainty of $\pm 3 \%$ in rotation which means that the method can be reliable even with $2 \sim 3^{\circ}$ error in rotation. Figure 2 presents the hardware setups utilized in the experiment.

\subsection{Hardware and Software Setup}

A set of five MPU-9250 IMUs was selected for the experiment, which all of them connected to a PC using a USB serial communication for recording the 3000 data in $30 \mathrm{~s}$ with $100 \mathrm{~Hz}$ sampling frequency. The data was recorded before and after the calibration. Finally, the method was implemented using MATLAB software in which, the bias vector $b$ and the matrix including soft iron, hard iron, and scale factor were estimated using UKF for each sensor, as described in Table 1. For evaluation of the results, the ellipsoid fitting method is used to fit the 3 -axis magnetometer points on a unit sphere. The results have shown that the calibration is well performed due to locating most of the points on the unit sphere. The data recording process was performed in 30 seconds. The number of magnetometer samples for each sensor is about 3000 . The comparison between uncalibrated and calibrated data with the proposed method has been shown in figures 3 to 5 . The data has been normalized to be located on the unit sphere. Figures 3-5 demonstrate the calibrated and uncalibrated magnetic field data for all the body-mounted sensors after normalization.

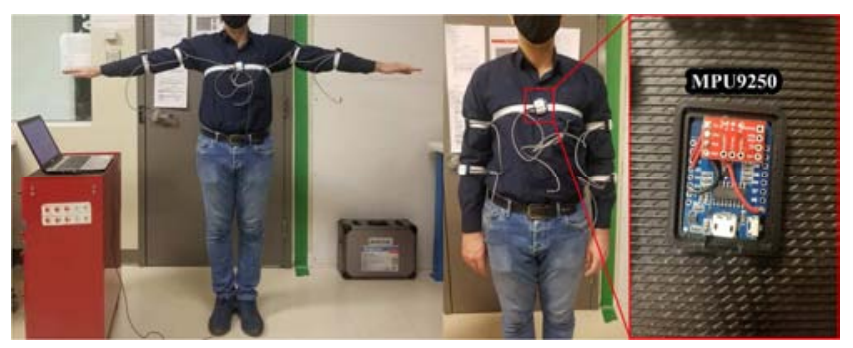

Figure 2. The experimental setup using five MPU9250 IMUs mounted on the body in the LASSENA laboratory.

\subsection{Results}

All the estimated states after performing the proposed method are considered in this part. It can be seen that the error parameters of calibration method are well estimated so, the calibrated data is located on the unit sphere with acceptable accuracy. The proposed method could show its functionality to be used for all the body-mounted sensors with just one single rotation. 
As the precise rotation needs extremely accurate turntable or robotic arms, the uncertainty of calibration is merely dependent on the rotation accuracy in the figure 1 .
Nevertheless, the paper's target is presenting the low-cost rotation method without any peripheral equipment.

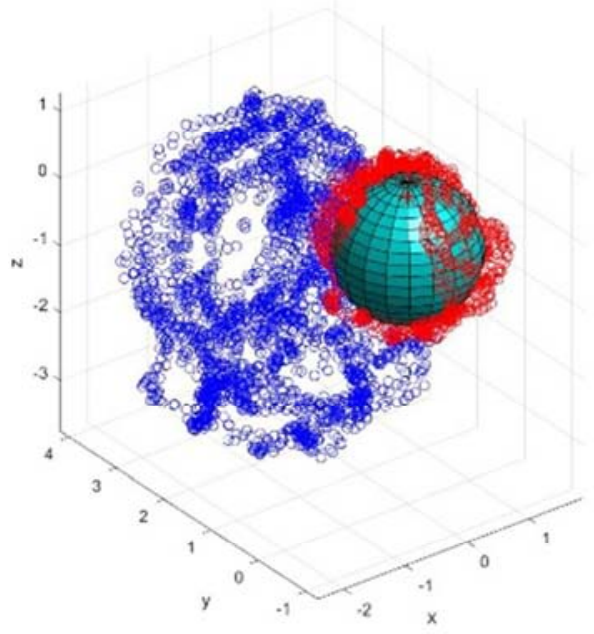

(a)

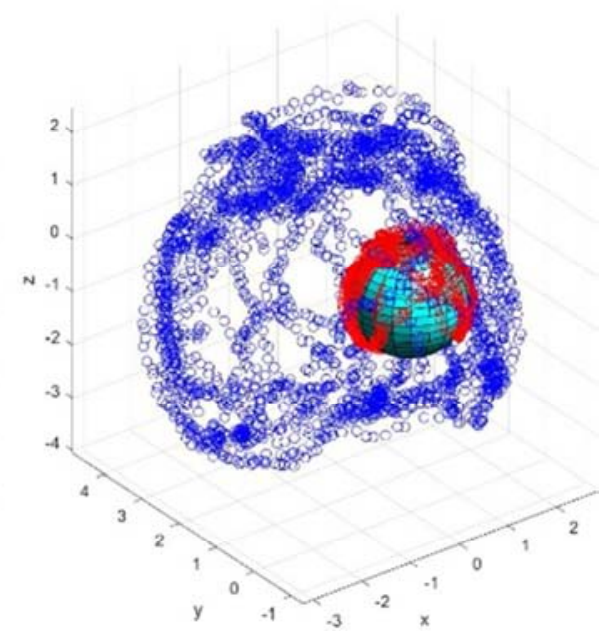

(b)

Figure 3. Comparison of calibrated and uncalibrated magnetic field data for (a): Left forearm sensor, and (b): Upper left arm sensor. (Blue color points: normalized uncalibrated data, Red color points: normalized calibrated data).

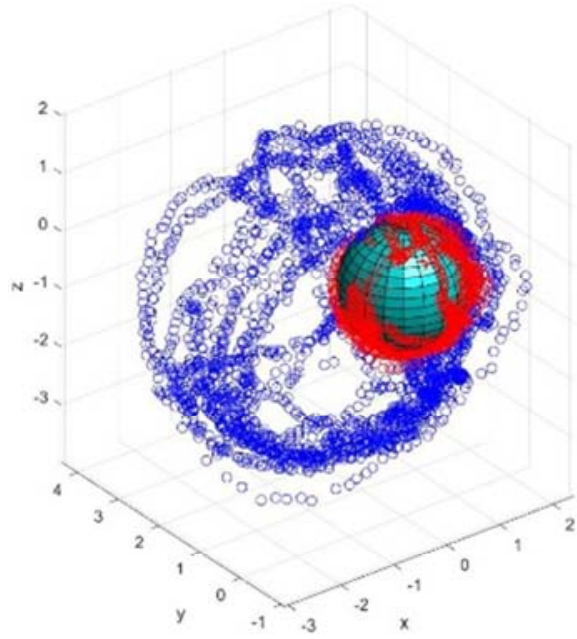

(a)

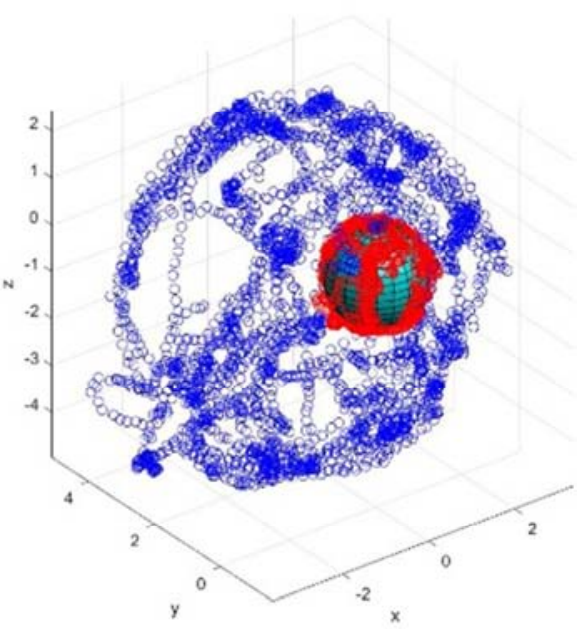

(b)

Figure 4. Comparison of calibrated and uncalibrated magnetic field data for (a): Upper right arm sensor, and (b): Right forearm sensor. (Blue color points: normalized uncalibrated data, Red color points: normalized calibrated data).

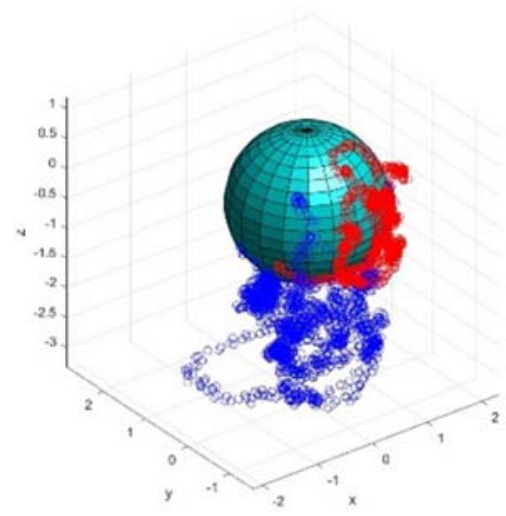

Figure 5. Comparison of calibrated and uncalibrated magnetic field data for the torso sensor. (Blue color points: normalized uncalibrated data, Red color points: normalized calibrated data).
Table 1. Estimated calibration parameters for bias vector and $A^{-1}$ Matrix for all sensors.

\begin{tabular}{llll}
\hline $\begin{array}{lll}\text { Sensor's } \\
\text { Position }\end{array}$ & Bias Vector & $\begin{array}{l}A^{\mathbf{1}} \text { Matrix (soft and hard iron } \\
\text { effects, scale factor) }\end{array}$ \\
\hline Torso & {$\left[\begin{array}{c}-53.0381 \\
286.5851 \\
-268.9892\end{array}\right]$} & {$\left[\begin{array}{ccc}0.1504 & -0.0226 & 0.0022 \\
-0.0226 & 0.1371 & 0.0085 \\
0.0022 & 0.0085 & 0.1455\end{array}\right]$} & {$\left[\begin{array}{ccc}0.1121 & 0.0022 & -0.0012 \\
0.0022 & 0.1120 & 0.0039 \\
-0.0012 & 0.0039 & 0.1064\end{array}\right]$} \\
Upper right arm & {$\left[\begin{array}{c}-33.7765 \\
396.7116 \\
-206.7799\end{array}\right]$} & {$\left[\begin{array}{ccc}0.0814 & 0.0032 & -0.0030 \\
0.0032 & 0.0872 & 0.0024 \\
-0.0030 & 0.0024 & 0.0766\end{array}\right]$} \\
Right forearm & {$\left[\begin{array}{c}-56.2727 \\
405.4633 \\
-221.8094\end{array}\right]$} & {$\left[\begin{array}{ccc}0.0981 & 0.0013 & -0.0028 \\
0.0013 & 0.0913 & 0.0002 \\
-0.0028 & 0.0002 & 0.0884\end{array}\right]$} \\
Upper left arm & {$\left[\begin{array}{c}-24.9804 \\
368.6235 \\
-190.7370\end{array}\right]$} & {$\left[\begin{array}{ccc}0.1645 & -0.0019 & -0.0053 \\
-0.0019 & 0.1457 & 0.0056 \\
-0.0053 & 0.0056 & 0.1336\end{array}\right]$} \\
\hline
\end{tabular}


With respect to this fact, the overall calibration of all the body-mounted magnetometers has proved the feasibility of the proposed method. Figures 6 and 9 represent the comparison of normalized magnetic field data for 30 seconds experiment for all five sensors. The normalized calibrated magnetometer outputs are approximately close to the unit value. Analysis of mean square error for all sensors is considered in the next subsection. Also, figure 10 shows the magnified view of the normalized calibrated magnetometer output for all body-mounted sensors.

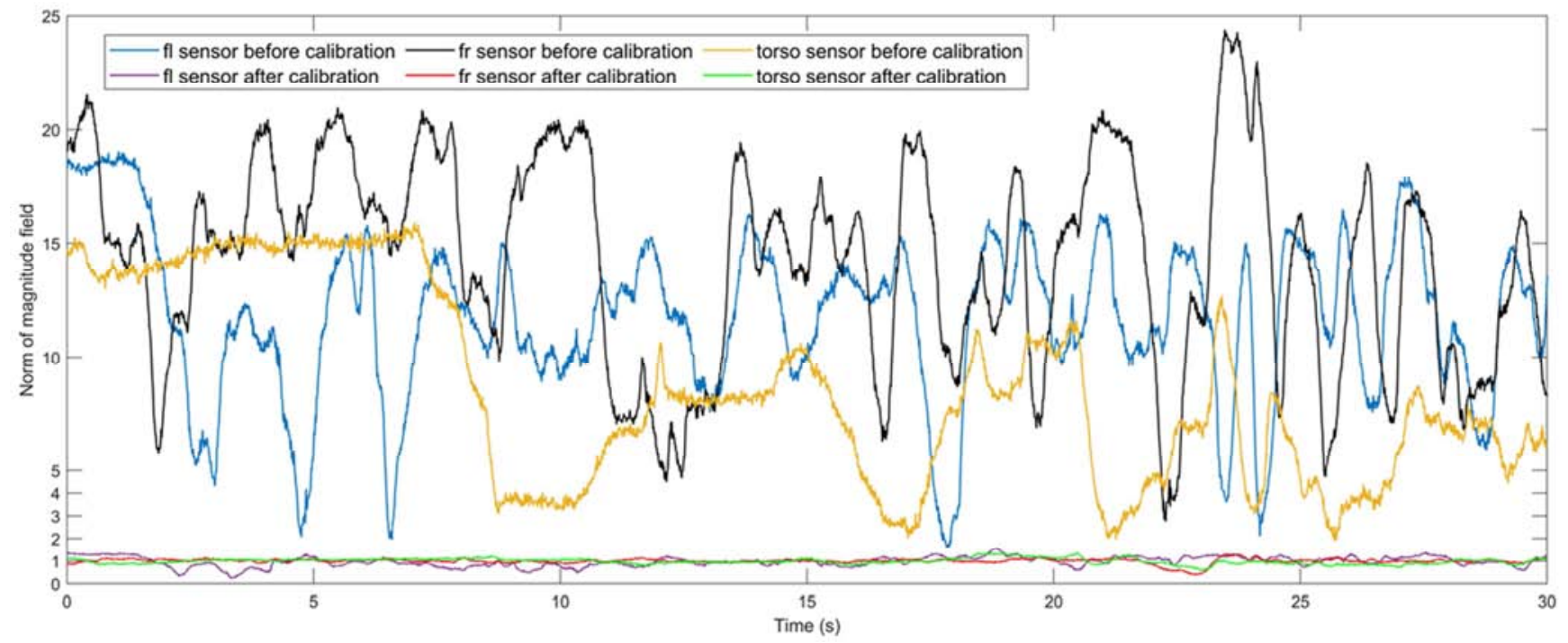

Figure 6. The magnetic field data during 30 seconds after and before calibration for left forearm (fl), right forearm (fr), and torso sensors.

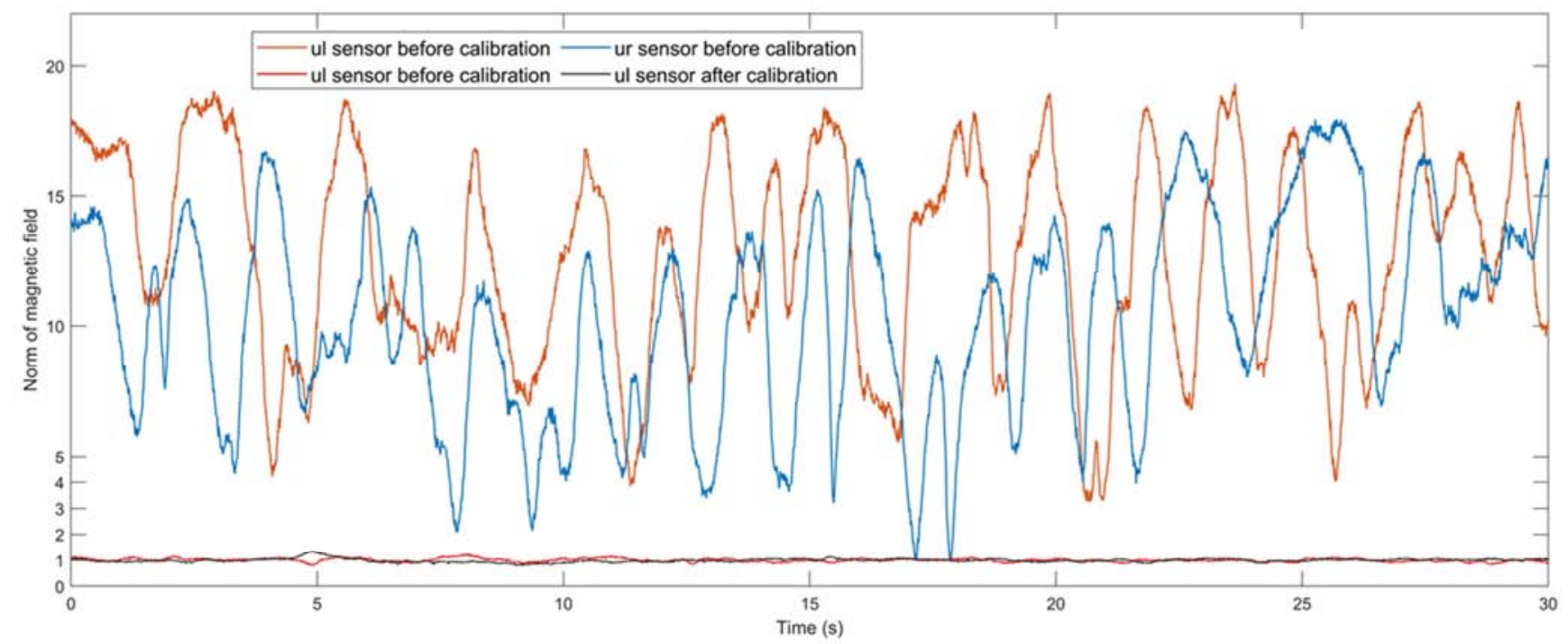

Figure 7. The magnetic field data during 30 seconds after and before calibration for upper left arm (ul), and upper right arm sensors.

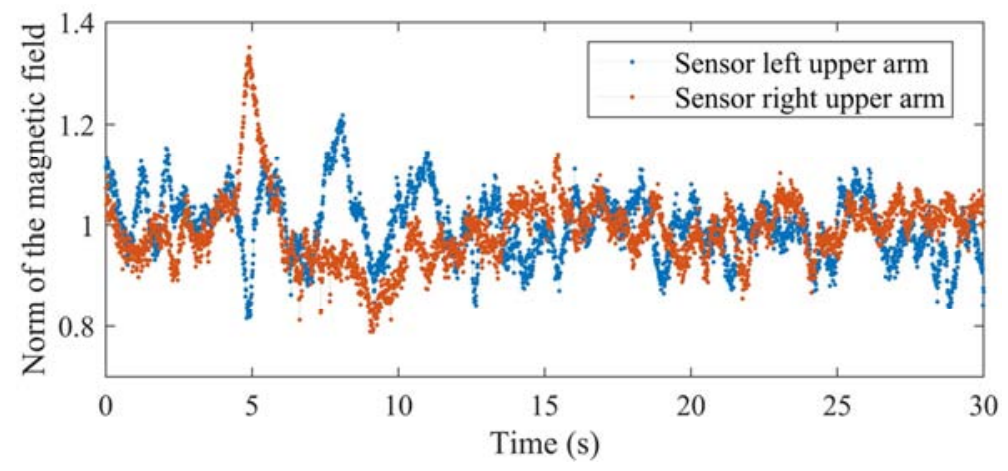

Figure 8. Normalized calibrated magnetic field for the left and right upper arm sensors. 




Figure 9. Normalized calibrated magnetic field for the torso, right, and left forearm sensors.

\subsection{Statistical Error Analysis}

The calibrated data in all five sensors are estimated and the Interquartile Range (IQR) method is performed to detect the outliers. This crucial step is done to make the measurements more meaningful. The outliers are defined as observations that fall below $Q_{1}-1.5(I Q R)$ or above $Q_{3}+1.5(I Q R)$, in which $Q_{1}$ and $Q_{2}$ are first quartile and third quartile, respectively. Based on this method, 1439 points have been detected and removed as outliers [27].

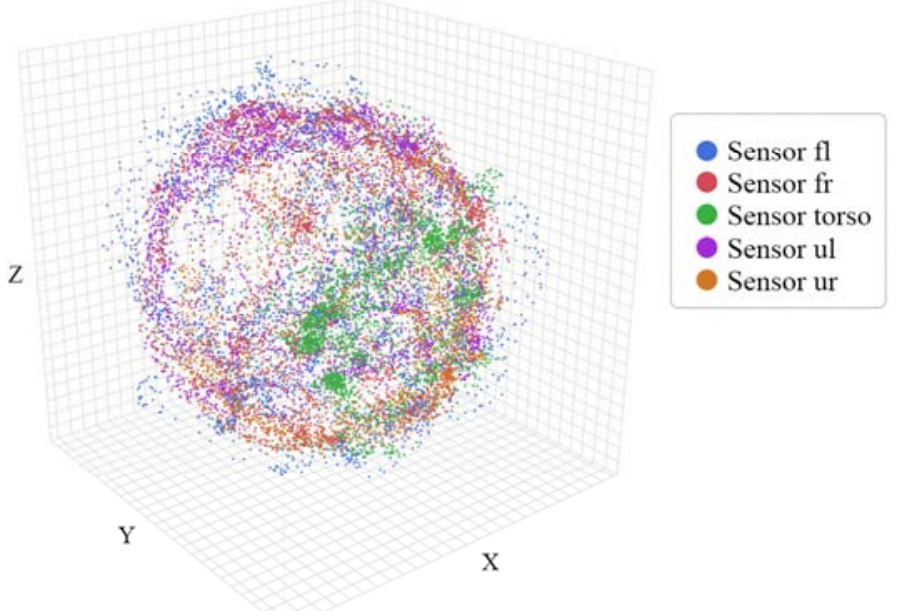

Figure 10. Rotation Trajectory for magnetometer set calibration of five upper body-mounted IMUs.

The left forearm sensor has the most dispersion around the reference which can be because of the sensor's precision, physical specification, and other environmental effects. The other investigation in accuracy of calibration has been performed by Mean Squared Error (MSE) analysis. The formula for obtaining this error analysis is pointed in Eqs. (40, 41) [28].

$$
\begin{gathered}
\operatorname{MSE}(\hat{\theta})=\operatorname{Var}_{\theta}(\hat{\theta})+\operatorname{Bias}(\hat{\theta}, \theta)^{2}, \\
\operatorname{MSE}(\hat{\theta})=E_{\theta}\left[(\hat{\theta}-\theta)^{2}\right],
\end{gathered}
$$

In which, $\hat{\theta}$ and $\theta$ are estimated and true unit reference as norm of each magnetometer data. Table 2 shows the MSE for each sensor.

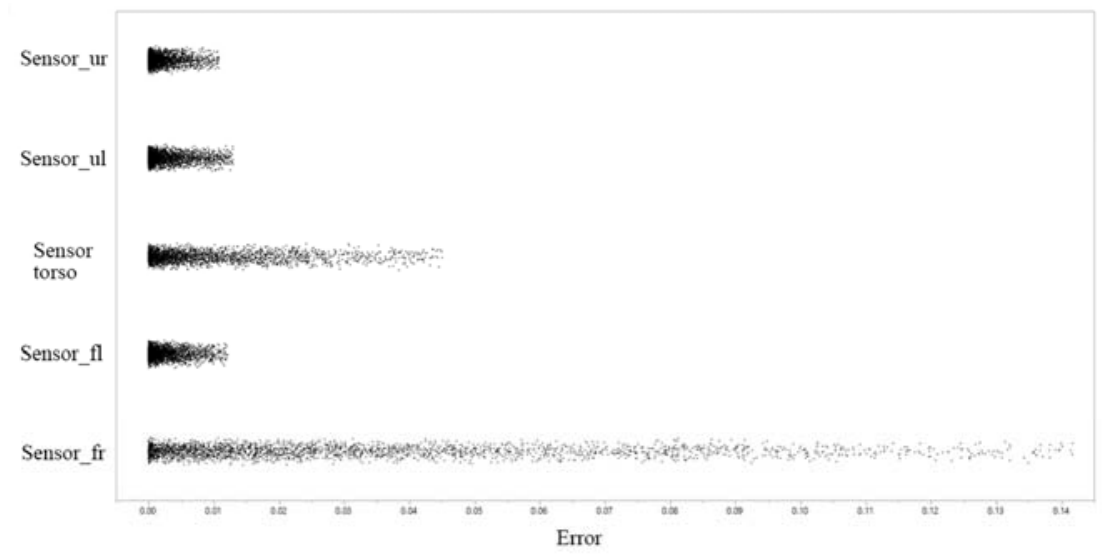

Figure 11. Error distribution graph for all five magnetometers. 
Table 2. MSE of all sensors for 3000 samples and $30 \mathrm{~s}$ experiment.

\begin{tabular}{lllll}
\hline $\begin{array}{l}\text { Left } \\
\text { forearm }\end{array}$ & $\begin{array}{l}\text { Right } \\
\text { forearm }\end{array}$ & Torso & $\begin{array}{l}\text { Upper left } \\
\text { arm }\end{array}$ & $\begin{array}{l}\text { Upper } \\
\text { right arm }\end{array}$ \\
\hline 0.03207 & 0.00237 & 0.00930 & 0.00277 & 0.00209 \\
\hline
\end{tabular}

It is demonstrated that the calibration error showed a negligible amount, which means that most of the magnetometer sensors have been calibrated with high precision, while mounted on the body. Also, figure 11 illustrates the distribution of error for each sensor. Upper right arm, upper forearm, and left forearm sensors are approximately calibrated as the same resolution, however; calibration accuracy of the torso and right forearm sensors have had more error desperation. The method prevents detaching the sensors one-by-one for calibration, especially in body motion capture applications, in which it can be time-wasting and difficult that all the sensors and other equipment be detached and calibrate separately.

\section{Conclusion}

In this paper, a 7-steps single-axis motion trajectory is designed to increase the observability of magnetometer error parameters. Because of an extreme nonlinear measurement sensor's model, the UKF system is designed to estimate the observable states of the calibration model. The most important contribution of this research is to increase the observability rank of complete system consists of 5 body mounted magnetometers without any multi-axis rotation trajectories, detachments, and expensive equipment. The observability rank of the system has been progressed from 40 to 57 with the proposed motion approach. The calibration effect of proposed methods verified has been assessed in an experiment in a real environment, and the results show that the body-mounted sensors are calibrated with MSE of about 0.01 in $30 \mathrm{~s}$.

There are multiple research gaps which can be covered by future investigations. Firstly, observability is still not full rank which means the system can show more accurate calibration results by improving the more precise state estimator or sensor modeling. Secondly, the uncertainty of estimated parameters can be studied in different environments like high and medium magnetic distortions, also, in the vicinity of different hard iron and soft iron materials. Thirdly, the calibration's influence in the results of motion capture and object tracking algorithms can deeply be surveyed. The impact of the proposed method in positioning and attitude results of various kinematic models can also be verified.

\section{Acknowledgements}

This work was supported by Director of LASSENA Laboratory (Laboratory of Space Technologies Embedded Systems, Navigation, and Avionic, Department of Electrical Engineering, École de Technologie supérieure Montreal, QC, Canada), Professor Rene Jr. Landry.

\section{References}

[1] Al-Fahoum, A. S., \& Abadir, M. S. (2018), "Design of a Modified Madgwick Filter for Quaternion-Based Orientation Estimation Using AHRS", Int. J. Comput. Electrical Eng., 10 (3), 174-186.

[2] Farhangian, F.; Landry, R., Jr, (2020) “Accuracy Improvement of Attitude Determination Systems Using EKF-Based Error Prediction Filter and PI Controller", Sensors, 20, 4055.

[3] C. M. N. Brigante, N. Abbate, A. Basile, A. C. Faulisi and S. Sessa, (2011) "Towards Miniaturization of a MEMS-Based Wearable Motion Capture System," in IEEE Transactions on Industrial Electronics, vol. 58, no. 8, pp. 3234-3241.

[4] Ozyagcilar, T. (2012), "Calibrating an ecompass in the presence of hard and soft-iron interference", Freescale Semiconductor Ltd, 1-17.

[5] Pang, Hongfeng, Ji Li, Dixiang Chen, Mengchun Pan, Shitu Luo, Qi Zhang, Feilu Luo (2013), "Calibration of three-axis fluxgate magnetometers with a nonlinear least square method", Measurement, vol. 46, pp. 1600-1606.

[6] Crassidis John L, Kok-Lam Lai, Richard R. Harman (2005), "Real-time attitude-independent three-axis magnetometer calibration", Journal of Guidance, Control, and Dynamics, vol. 28, pp. 115-20.

[7] Wu Y, W. Shi (2015), "On calibration of three-axis magnetometer”, IEEE Sensors Journal, vol. 15, pp. 6424-31.

[8] M. Kok, J. D Hol, T. B. Schön, F. Gustafsson (2012), H. Luinge, "Calibration of a magnetometer in combination with inertial sensors", 15th International Conference on Information Fusion, Singapore, pp. 787-793.

[9] Riwanto, Bagus Adiwiluhung, Tuomas Tikka, Antti Kestila, Jaan Praks (2017), "Particle swarm optimization with rotation axis fitting for magnetometer calibration", IEEE Transactions on Aerospace and Electronic Systems, vol. 53, pp. 1009-22.

[10] Beravs Tadej, Samo Begus, Janez Podobnik, Marko Munih (2014), "Magnetometer calibration using Kalman filter covariance matrix for online estimation of magnetic field orientation", IEEE Transactions on Instrumentation and Measurement, vol. 63, pp. 2013-20.

[11] Wu Yuanxin, Danping Zou, Peilin Liu, Wenxian Yu (2018), "Dynamic magnetometer calibration and alignment to inertial sensors by Kalman filtering" IEEE Transactions on Control Systems Technology, vol. 26, pp. 716-23.

[12] Yadav, Nagesh, and Chris Bleakley (2016), "Fast calibration of a 9-DOF IMU using a 3 DOF position tracker and a semi-random motion sequence", Measurement, vol. 90, pp. 192-98.

[13] Han Ke, He Han, Zhifeng Wang, Feng Xu (2016), "Extended Kalman filter-based gyroscope aided magnetometer calibration for consumer electronic devices", IEEE Sensors Journal, pp. 1-1.

[14] Soken Halil Ersin, Chingiz Hajiyev (2012), "UKF-based reconfigurable attitude parameters estimation and magnetometer calibration", IEEE Transactions on Aerospace and Electronic Systems, vol. 48, pp. 2614-27. 
[15] Rui Zhang, Fabian Hoflinger, Leonhard M. Reind (2014), "Calibration of an IMU using 3-D rotation platform", IEEE Sensors Journal, vol. 14, pp. 1778-87.

[16] Salehi, S., Mostofi, N. and Bleser, G., (2012), A practical in-field magnetometer calibration method for IMUs, In Proceedings of the IROS Workshop on Cognitive Assistive Systems: Closing the Action-Perception Loop (pp. 39-44).

[17] Pang, Hongfeng, Dixiang Chen, Mengchun Pan, Shitu Luo, Qi Zhang, Ji Li, and Feilu Luo (2013), "A new calibration method of three-axis magnetometer with nonlinearity suppression", IEEE Transactions on Magnetics, vol. 49, pp. 5011-15.

[18] Renaudin Valérie, Muhammad Haris Afzal, Gérard Lachapelle (2010), "Complete triaxis magnetometer calibration in the magnetic domain”, Journal of Sensors, pp. 1-10.

[19] Soken, H. E., and Sakai, S. I., (2020). Attitude estimation and magnetometer calibration using reconfigurable TRIAD+ filtering approach. Aerospace Science and Technology, 99, p. 105754 .

[20] Opromolla, R. Magnetometer Calibration for Small Unmanned Aerial Vehicles Using Cooperative Flight Data. Sensors 2020, 20,538 .

[21] Wang, Y., Sheng, T., He, L. and Cheng, Z. (2020) Calibration Method of Magnetometer Based on BP Neural Network. Journal of Computer and Communications, 8, 31-41.
[22] N. Nguyen and P. Müller, "A Novel Magnetometer Calibration Approach with Artificial Data," 2020 27th Saint Petersburg International Conference on Integrated Navigation Systems (ICINS), Saint Petersburg, Russia, 2020, pp. 1-4.

[23] Yang, Y., Xiao, X., \& Liao, W. (2016, January), "Magnetometer Calibration Algorithm Based on Analytic Geometry Transform", In 2015 2nd International Forum on Electrical Engineering and Automation (IFEEA 2015). Atlantis Press.

[24] M. Zhu, Y. Wu and W. Yu, (2019) "An Efficient Method for Gyroscope-Aided Full Magnetometer Calibration," in IEEE Sensors Journal, vol. 19, no. 15, pp. 6355-6361.

[25] Alberto Isidori (1997), Nonlinear Control Systems, Springer-Verlag, Berlin, Heidelberg.

[26] Goshen-Meskin, D. and I. Bar-Itzhack (1990), “Observability Analysis of piece-wise constant systems with application to inertial navigation", 29th IEEE Conference on Decision and Control, vol. 2, pp. 821-826.

[27] Tukey, John W (1977), Exploratory data analysis, pp. 44-45.

[28] Thomas H. Wonnacott, Ronald J. Wonnacott (1990), Introductory Statistics, pp. 137-140. 